\title{
BMJ Open Prevalence and associated factors of diabetic retinopathy in Beijing, China: a cross-sectional study
}

\author{
Jing Cui, ${ }^{1}$ Ji-Ping Ren, ${ }^{1}$ Dong-Ning Chen, ${ }^{1}$ Zhong Xin, ${ }^{2}$ Ming-Xia Yuan, ${ }^{2}$ Jie Xu, ${ }^{3}$ \\ Qi-Sheng You, ${ }^{3}$ Jin-Kui Yang ${ }^{2}$
}

To cite: Cui J, Ren J-P, Chen D-N, et al. Prevalence and associated factors of diabetic retinopathy in Beijing, China: a cross-sectional study. BMJ Open 2017;7:e015473. doi:10.1136/ bmjopen-2016-015473

- Prepublication history for this paper is available online. To view these files please visit the journal online (http://dx.doi. org/10.1136/bmjopen-2016015473).

Received 7 December 2016 Revised 6 May 2017 Accepted 24 May 2017

\section{CrossMark}

${ }^{1}$ Department of Physical Examination, Beijing Tongren Hospital, Capital Medical University, Beijing, China ${ }^{2}$ Department of Endocrinology, Beijing Tongren Hospital, Capital Medical University, Beijing, China

${ }^{3}$ Beijing Institute of Ophthalmology, Beijing Tongren Eye Center, Beijing Tongren Hospital, Capital Medical University, Beijing, China

Correspondence to

Dr. Jin-Kui Yang;

jkyang@ccmu.edu.cn

\section{ABSTRACT}

Objectives The study aimed to determine the exact risk factors for diabetic retinopathy (DR) in the Chinese population using a cohort of 17985 individuals from Beijing, China.

Design Cross-sectional study.

Setting A hospital.

Participants 17985 individuals from Beijing, China. Primary and secondary outcome measures This was a cross-sectional study of permanent residents from the Changping area (Beijing, China) recruited from July 2010 to March 2011 and from March 2014 to February 2015 during a routine health examination at the Tongren Hospital of Beijing. Eye examinations were conducted by experienced ophthalmologists. Medical history, height, weight, body mass index (BMI) and blood pressure were recorded. Routine laboratory examinations were performed.

Results The prevalence of DR was $1.5 \%$ in the general study population and $8.1 \%$ among individuals with diabetes. Compared with the non-DR group, individuals in the DR group in the diabetes population had longer disease duration, higher systolic blood pressure (SBP), fasting plasma glucose (FPG) and uric acid (UA) (in men) and lower UA (in women) (all $p<0.05$ ). The multivariate analysis showed that disease duration $(p<0.001)$, BMI $(p=0.046), \operatorname{SBP}(p=0.012)$, creatinine clearance rate (CCR) $(p=0.014)$, UA $(p=0.018)$ and FPG $(p<0.001)$ were independently associated with $\mathrm{DR}$ in patients with diabetes.

Conclusion The prevalence of DR was $8.1 \%$ among patients with diabetes. Disease duration, BMI, SBP, CCR, UA and FPG were independently associated with DR.

\section{INTRODUCTION}

Diabetic retinopathy (DR) is an important cause of vision impairment and blindness. ${ }^{1}$ With the increasing prevalence of diabetes in the world, ${ }^{1}$ DR has become a disease that severely threatens public health. Vision deterioration can be prevented and the risk of blindness can be reduced if fundus screening and early intervention are performed in patients with diabetes. ${ }^{1}$

A systematic review based on the global census of population from 1980 to 2008 has

\section{Strengths and limitations of this study}

- The present study identified risk factors of diabetic retinopathy (DR) in a large sample of patients with diabetes. The results could help identify patients at high risk of DR and allow preventive measures to be taken early.

- There is a possibility of a selection bias because the recruited individuals were visiting the hospital for a routine health examination.

- Not all subjects underwent an oral glucose tolerance test, which could underestimate the prevalence of diabetes.

- Compared with multiview fundus examination, single-view fundus examination may underestimate the prevalence of DR.

- Some individuals were excluded because of other eye diseases.

shown that the prevalence of DR is $34.6 \% .^{2}$ In fact, there are great differences in the prevalence of DR among various countries. Specifically, the highest prevalence of DR is $49.6 \%$ in African groups in the USA, while the lowest is $19.9 \%$ in Asian groups in host countries; in between is China with $25.1 \%{ }^{2}$ A systematic review of studies published between 1986 and 2009 suggested that the prevalence of DR in mainland China was $23 \%{ }^{3}$

Diabetes duration, blood glucose and blood pressure are widely accepted risk factors for $\mathrm{DR},{ }^{2}$ and some studies indicated that blood lipids, ${ }^{2}$ body mass index (BMI) and renal function ${ }^{2}$ also affect the occurrence of DR. Since the reported prevalence of DR in Chinese individuals is low despite the increasing prevalence of diabetes (but regional variations do exist) and considering the balance of disease and economic benefits, specific screening strategies have to be developed according to the actual situation in China. In China, healthcare is available to treat diabetic complications (such as retinopathy, foot 
ulcers, kidney diseases, etc) but there is no screening programme for diabetes. Therefore, most patients become aware of their diabetic status once serious complications occur, hence the importance of a screening programme.

Therefore, in order to determine the exact risk factors for DR in the Chinese population, a cohort of 17985 individuals in Beijing (China) was recruited. These individuals underwent screening for diabetes and a survey for the prevalence of DR. This study aimed to analyse the risk factors for DR.

\section{METHODS}

\section{Study design and subjects}

This was a cross-sectional study of permanent residents from the Changping area (Beijing, China) recruited from July 2010 to March 2011 and from March 2014 to February 2015 during a routine health examination at the Tongren Hospital of Beijing. The inclusion criterion was being 18-79 years of age. The exclusion criteria were: (1) reluctant respondents; (2) did not complete the questionnaire, physical examination, oral glucose tolerance test (OGTT) or blood tests; (3) cataract, glaucoma or any other eye diseases; or (4) fundus examination could not be completed for any reason.

The permanent resident population of the Changping area (suburb) of Beijing was 1660 500. A total of 8155 people were selected and invited to participate in the study by using a multistage, stratified random sampling method. During the study periods, 2551 individuals who participated in the Changping Epidemiological Study and whose fasting plasma glucose was $>5.6 \mathrm{mmol} / \mathrm{L}$ completed the OGTT and ophthalmic examination. Among 15671 individuals receiving routine health check-up, 237 individuals were excluded for eye diseases, and 15434 people were included in this analysis. Therefore, the overall study population (from the Changping Epidemiological Study and from health examinations) was 17985 individuals.

The present study was approved by the ethics committee of the Tongren Hospital of Beijing. Each subject provided a written informed consent.

\section{Diagnostic criteria}

The diagnosis criteria for diabetes were: (1) fasting plasma glucose (FPG) $\geq 7.0 \mathrm{mmol} / \mathrm{L}$; (2) history of diabetes; (3) taking antidiabetes medication; or (4) OGTT results consistent with the criteria of the 1997 American Diabetes Association (ADA). ${ }^{4}$ According to the Early Treatment Diabetic Retinopathy Study, those with the following lesions in fundus image were diagnosed with DR: (1) microaneurysms; (2) haemorrhage; (3) hard exudates; (4) cotton wool spots; (5) retinal vein beaded change; (6) microvascular abnormalities in the retina; and/or (7) neovascularisation.

\section{Fundus examination}

Eye examinations were conducted by experienced ophthalmologists. Mydriasis of both eyes was conducted and a Topcon TRC-NW7SF fundus camera (Topcon, Tokyo, Japan) was used to capture $45^{\circ} \mathrm{C}$ colour digital images of the fundus of both eyes. A double-blind diagnosis was performed by two ophthalmologists from the Eye Institute of the Affiliated Beijing Tongren Hospital of Capital Medical University. In case of disagreement, a third ophthalmologist was consulted.

\section{Data collection and laboratory examinations}

Medical history, height and weight were recorded. BMI was calculated as weight in kilograms divided by height in metres squared. A standard mercury sphygmomanometer was used to measure the blood pressure three times in the sitting position after 5 min rest; the average value was used for analysis.

Fasting antecubital venous blood was sampled to measure FPG. If FPG was $\geq 5.6 \mathrm{mmol} / \mathrm{L}$, a standard $75 \mathrm{~g}$ glucose OGTT was performed within 8-10 hours. All measurements were performed in laboratories submitted to the quality control process of the Chinese Ministry of Health. A glucose oxidase method was used for the measurement of blood glucose. A Hitachi 7600 analyzer was used to detect creatinine, uric acid (UA) and blood lipids (total cholesterol (TC), triglycerides (TG), high-density lipoprotein cholesterol (HDL-C) and low-density lipoprotein cholesterol (LDL-C)). Creatinine clearance rate $(\mathrm{CCR}) \quad(\mathrm{mL} / \mathrm{min})=((140-$ age $) \times$ weight $(\mathrm{kg})) /($ creatinine $(\mu \mathrm{mol} / \mathrm{L}) \times 0.82$ (men) or 0.85 (women)). All blood samples were centrally analysed within 24 hours.

\section{Statistical analysis}

Continuous data were presented as mean $\pm \mathrm{SD}$, and categorical data were presented as frequencies. Normally distributed continuous data were analysed using the independent t-test, while the rank sum test was used for non-normally distributed data. The $\chi^{2}$ test was used for categorical data. After adjusting for age and gender using binary logistic regression, the evaluation of ORs and $95 \% \mathrm{CI}$ of the risk factors for DR was performed. In the binary logistic regression analysis, the continuous variables were FPG, UA, TC, TG, LDL-C and HDL-C. The patients were grouped as diabetes, pre-diabetes and normal glucose tolerance (NGT), according to the 1997 ADA guidelines. ${ }^{4}$ For the multivariate analyses performed in patients with diabetes and pre-diabetes, the continuous data were transformed into categorical data for the logistic regression: (1) age was divided into 10-year groups; (2) blood pressure was divided into $10 \mathrm{~mm} \mathrm{Hg}$ groups; (3) the course of the disease was divided into three groups: $<5,5-9$ and $>9$ years; (4) BMI: $<24$ and $\geq 24 \mathrm{~kg} / \mathrm{m}^{2}$; (5) CCR $<90 \mathrm{~mL} /$ min (abnormal) and CCR $\geq 90 \mathrm{~mL} / \mathrm{min}$ (normal); (6) abdominal obesity: men, waist circumference $\geq 85 \mathrm{~cm}$, 
Table 1 Characteristics of the study population

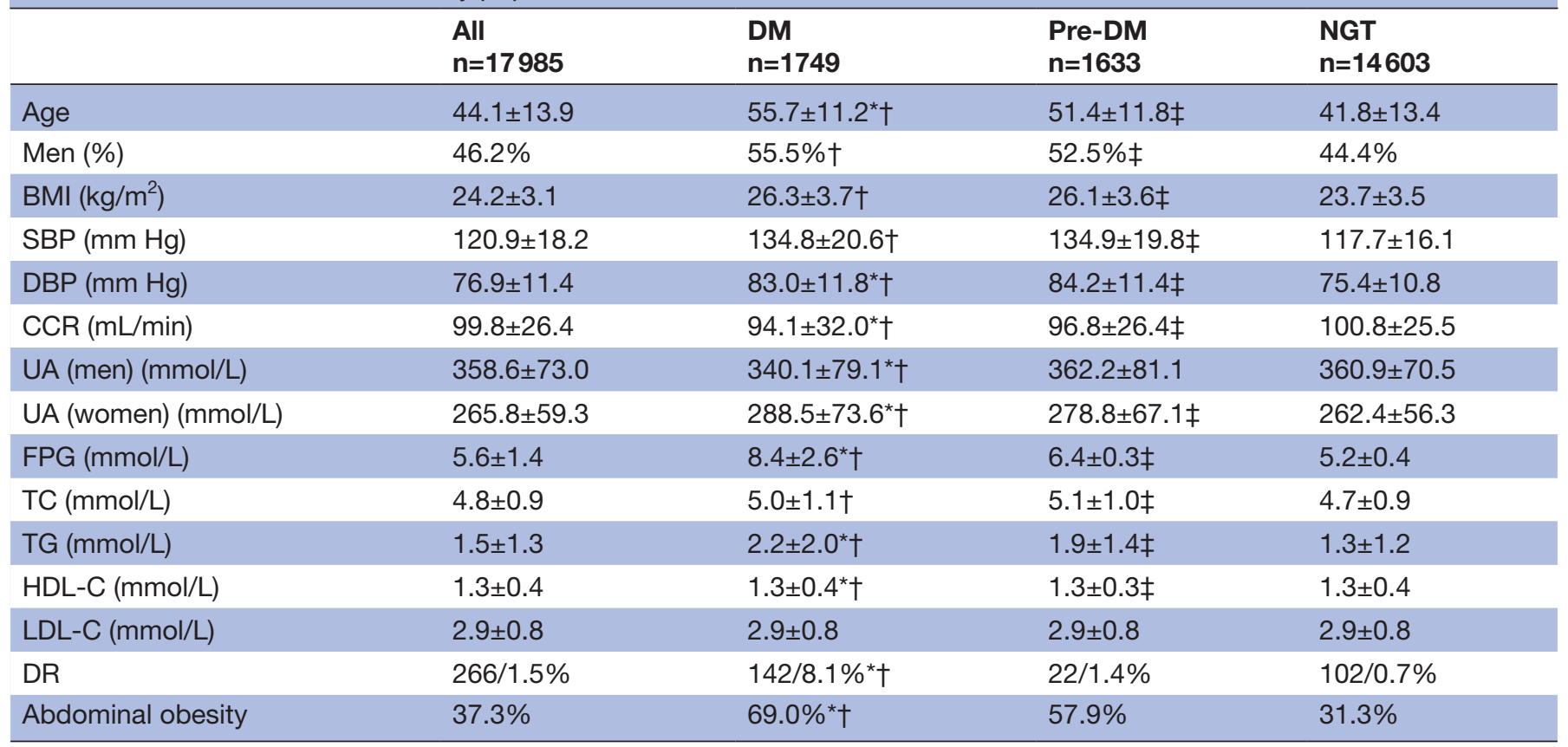

${ }^{*}$ DM vs pre-DM, $p<0.05$.

†DM vs NGT, $p<0.05$.

†Pre-DM vs NGT, $p<0.05$.

BMI, body mass index; CCR, creatinine clearance rate; DBP, diastolic blood pressure; DM, diabetes mellitus; DR, diabetic retinopathy; FPG, fasting plasma glucose; HDL-C, high-density lipoprotein cholesterol; LDL-C, low-density lipoprotein cholesterol; NGT, normal glucose tolerance; SBP, systolic blood pressure; TC, total cholesterol; TG, triglycerides; UA, uric acid.

women, waist circumference of $\geq 80 \mathrm{~cm}$, or waist-to-hip ratio $\geq 0.93$. SPSS V.22.0 for Windows (IBM) was used for statistical analysis. Two-sided p-values $<0.05$ were considered statistically significant.

\section{RESULTS}

\section{Characteristics of the study population}

All patients had type 2 diabetes, based on medical history, patient age and drug history. The average age of the overall study population (17985 individuals) was $44.1 \pm 13.9$ years (range, 18-79 years), and 1749 people were identified to be with diabetes. The average age of the patients with diabetes was $55.7 \pm 11.2$ years. Age, BMI, blood pressure, FPG, TC and TG in the diabetes group were all significantly higher than in the non-diabetes group, while the CCR in the diabetes group was lower than that in the non-diabetes group (table 1).

\section{Prevalence and characteristics of DR}

There were 261 patients with DR in the general study population, for a prevalence of $1.5 \%$. There were 141 patients with DR in patients with diabetes, for a prevalence of $8.1 \%$ (the prevalence of known diabetes was $11.8 \%$, and the prevalence of newly diagnosed diabetes was $2.8 \%$ ). There were 120 patients with DR in individuals without diabetes, for a prevalence of $0.7 \%$ (cases with abnormal OGTT or increased FPG were $1.4 \%$, and cases with normal OGTT or normal FPG were $0.7 \%$ ).
Compared with the non-DR group, individuals in the DR group in the diabetes population had longer disease duration, higher systolic blood pressure (SBP), FPG, and UA (in men) and lower UA (in women). There was no significant difference in CCR, TC, TG, HDL-C and LDL-C levels between the two groups (table 2).

\section{Multivariate analysis of the risk of DR among patients with diabetes}

The risk factors for retinopathy were analysed in the patients with diabetes. The multivariate analysis showed that disease duration (OR 1.74, 95\% CI 1.37 to 2.20, $\mathrm{p}<0.001$ ), BMI $\geq 24 \mathrm{~kg} / \mathrm{m}^{2}$ (OR $1.58,95 \%$ CI 1.01 to 2.48 , $\mathrm{p}=0.046$ ), SBP (for $10 \mathrm{~mm} \mathrm{Hg}$ increases; OR $1.13,95 \%$ CI 1.03 to $1.24, \mathrm{p}=0.012$ ), CCR $<90 \mathrm{~mL} / \mathrm{min}$ (OR 1.61, 95\% CI 1.10 to $2.36, \mathrm{p}=0.014)$, UA (OR $0.997,95 \%$ CI 0.995 to $0.999, \mathrm{p}=0.018)$ and FPG (OR 1.22, 95\% CI 1.15 to $1.29, \mathrm{p}<0.001$ ) were independently associated with $\mathrm{DR}$ (table 3).

\section{Multivariate analysis of the risk of DR among patients with pre-diabetes}

The risk factors for retinopathy were analysed in the individuals with pre-diabetes. The multivariate analysis showed that SBP (for $10 \mathrm{~mm} \mathrm{Hg}$ increases; OR 1.14, $95 \%$ CI 1.06 to $1.21, \mathrm{p}<0.001)$ and FPG (OR 1.38, 95\% CI 1.33 to $1.44, \mathrm{p}<0.001$ ) were independently associated with DR. 
Table 2 Comparison of the characteristics between subjects with or without DR

\begin{tabular}{|c|c|c|c|}
\hline \multicolumn{3}{|c|}{\begin{tabular}{lll} 
DR \\
\cline { 2 - 2 }
\end{tabular}} & \multirow[t]{2}{*}{ p Value } \\
\hline & Negative (1608) & Positive (141) & \\
\hline Age & $55.9 \pm 11.3$ & $57.1 \pm 10.3$ & 0.202 \\
\hline Men (\%) & $55.5 \%$ & $55.6 \%$ & 0.979 \\
\hline Diabetes duration & $4.5 \pm 4.2$ & $7.1 \pm 4.6$ & $<0.001^{*}$ \\
\hline $\operatorname{BMI}\left(\mathrm{kg} / \mathrm{m}^{2}\right)$ & $26.3 \pm 3.7$ & $25.9 \pm 2.9$ & 0.090 \\
\hline $\mathrm{SBP}(\mathrm{mm} \mathrm{Hg})$ & $134.4 \pm 20.3$ & $139.7 \pm 23.2$ & $0.007^{*}$ \\
\hline $\mathrm{DBP}(\mathrm{mm} \mathrm{Hg})$ & $82.9 \pm 11.8$ & $82.6 \pm 12.2$ & 0.757 \\
\hline $\mathrm{CCR}(\mathrm{mL} / \mathrm{min})$ & $94.5 \pm 32.7$ & $90.3 \pm 27.4$ & 0.132 \\
\hline UA (men) (mmol/L) & $341.8 \pm 79.6$ & $321.4 \pm 72.3$ & $0.029^{*}$ \\
\hline UA (women) (mmol/L) & $290.7 \pm 73.7$ & $263.5 \pm 68.9$ & $0.005^{*}$ \\
\hline FPG (mmol/L) & $8.2 \pm 2.5$ & $10.2 \pm 3.4$ & $<0.001^{*}$ \\
\hline $\mathrm{TC}(\mathrm{mmol} / \mathrm{L})$ & $5.0 \pm 1.1$ & $5.1 \pm 1.2$ & 0.662 \\
\hline $\mathrm{TG}(\mathrm{mmol} / \mathrm{L})$ & $2.2 \pm 2.1$ & $2.2 \pm 2.2$ & 0.740 \\
\hline $\mathrm{HDL}-\mathrm{C}(\mathrm{mmol} / \mathrm{L})$ & $1.3 \pm 0.4$ & $1.3 \pm 0.3$ & 0.486 \\
\hline LDL-C (mmol/L) & $2.9 \pm 0.8$ & $2.9 \pm 0.9$ & 0.967 \\
\hline Abdominal obesity (\%) & $68.9 \%$ & $70.2 \%$ & 0.744 \\
\hline
\end{tabular}

${ }^{*} \mathrm{p}<0.05$.

$\mathrm{BMI}$, body mass index; CCR, creatinine clearance rate; DBP, diastolic blood pressure; DM, diabetes mellitus; DR, diabetic retinopathy; FPG, fasting plasma glucose; HDL-C, high-density lipoprotein cholesterol; LDL-C, low-density lipoprotein cholesterol; NGT, normal glucose tolerance; SBP, systolic blood pressure; TC, total cholesterol; TG, triglycerides; UA, uric acid.

Table 3 Univariate and multivariate analyses of factors associated with DR in patients with diabetes

\begin{tabular}{|c|c|c|c|c|}
\hline \multirow[b]{2}{*}{ Variables } & \multicolumn{2}{|c|}{ Univariate } & \multicolumn{2}{|c|}{ Multivariate } \\
\hline & p Value & OR $(95 \% \mathrm{Cl})$ & p Value & OR $(95 \% \mathrm{Cl})$ \\
\hline Age (category) & 0.077 & $1.136(0.972$ to 1.328$)$ & 0.270 & $0.882(0.706$ to 1.102$)$ \\
\hline Duration (category) & $<0.001$ & 1.503 (1.201 to 1.882$)$ & $<0.001$ & 1.735 (1.368 to 2.202$)$ \\
\hline BMI (category) & 0.593 & $1.116(0.747$ to 1.666$)$ & 0.046 & 1.579 (1.007 to 2.475$)$ \\
\hline DBP (category) & 0.644 & 0.965 (0.828 to 1.124$)$ & 0.085 & $0.842(0.692$ to 1.024$)$ \\
\hline CCR (category) & 0.059 & 1.395 (0.987 to 1.972$)$ & 0.014 & 1.613 (1.103 to 2.358$)$ \\
\hline UA & 0.001 & 0.996 (0.994 to 0.998$)$ & 0.018 & 0.997 (0.995 to 0.999) \\
\hline FPG & $<0.001$ & $1.215(1.154$ to 1.279$)$ & $<0.001$ & $1.220(1.152$ to 1.291$)$ \\
\hline LDL-C & 0.967 & $1.004(0.819 .1 .232)$ & 0.363 & $0.767(0.434$ to 1.357$)$ \\
\hline Abdominal obesity (category) & 0.581 & $1.111(0.764$ to 1.618$)$ & 0.486 & $1.198(0.721$ to 1.992$)$ \\
\hline
\end{tabular}

The continuous data were transformed into categorical data: (1) age was divided into 10-year groups; (2) blood pressure was divided into $10 \mathrm{~mm} \mathrm{Hg}$ groups; (3) the course of the disease was divided into three groups: $<5,5-9$ and $>9$ years; (4) BMl: $<24 \mathrm{~kg} / \mathrm{m}^{2}(\mathrm{normal}),>24 \mathrm{~kg} / \mathrm{m}^{2}$ (overweight); (5) CCR $<90 \mathrm{~mL} / \mathrm{min}$ (abnormal), CCR $>90 \mathrm{~mL} / \mathrm{min}$ (normal); (6) abdominal obesity: men, waist circumference $>85 \mathrm{~cm}$, women, waist circumference of $>80 \mathrm{~cm}$, or waist-to-hip ratio $>0.93$.

$\mathrm{BMI}$, body mass index; CCR, creatinine clearance rate; DBP, diastolic blood pressure; DR, diabetic retinopathy; FPG, fasting plasma glucose; HDL-C, high-density lipoprotein cholesterol; LDL-C, low-density lipoprotein cholesterol; SBP, systolic blood pressure; TC, total cholesterol; TG, triglycerides; UA, uric acid. 


\section{DISCUSSION}

The prevalence of DR in Chinese individuals is low despite the increasing prevalence of diabetes (but regional variations do exist). This study aimed to determine the exact risk factors for DR in the Chinese population using a cohort of 17985 individuals from Beijing, China. The prevalence of DR was $1.5 \%$ in the general study population or $8.1 \%$ among patients with diabetes. Disease duration, BMI, SBP, CCR and FPG were independently associated with DR.

In the present study, the prevalence of DR in the patients with diabetes was $8.1 \%$, which was significantly lower than in other countries such as Norway $(34.6 \%))^{5}$ the USA $(28.5 \%),{ }^{6}$ Iceland $(25.2 \%)^{7}$ and Africa $(30.2 \%-$ $31.6 \%),{ }^{8}$ and also lower than the worldwide prevalence $(34.6 \%) .^{2}$ Studies indicate that ethnic differences are the main factors leading to differences of the prevalence among different populations after adjusting for general risk factors, ${ }^{9}$ and still, the prevalence of DR in Asians remains the lowest, at $19.9 \%{ }^{2}$

Nevertheless, some studies have suggested that South Asians are more likely to have DR compared with white Europeans, but Asians with DR are younger, the course of disease is shorter and blood pressure and FPG are higher. ${ }^{10}$ Although there is no ethnic difference among Asian countries, ${ }^{11}$ the prevalence observed in the present study is still far lower than in other Asian countries such as Bangladesh $(21.6 \%),{ }^{12}$ India $(21.7 \%)^{13}$ and Singapore $(35 \%),{ }^{14}$ and even lower than the results of other mainland cities such as Shanghai $(22.9 \%),{ }^{15}$ Beijing $(37.1 \%)^{16}$ and Handan $(43.1 \%),{ }^{17}$ but it is similar to the prevalence observed in a study in Shanghai $(9.6 \%) .{ }^{18}$ These differences among studies may be caused by the sample size, the type of study population, age, course of disease, the average levels of various variables and different methods for fundus examination.

This study confirmed the commonly accepted risk factors for DR such as the course of diabetes, SBP and FPG. ${ }^{2}$ In the present study, the prevalence of DR was $11.8 \%$ in individuals with a known history of diabetes and $2.8 \%$ in individuals with newly diagnosed diabetes. In addition, disease duration was independently associated with DR, as supported by a previous study. ${ }^{19}$ Of course, there is a high probability that undiagnosed patients before study participation were at the beginning of the disease, before onset of diabetic symptoms. Therefore, a less severe diabetes should be associated with fewer complications such as DR. Patients without known diabetes but with high suspicion or diagnosis of DR on fundus examination should undergo screening for diabetes. In addition, the present study found that the risk of DR increased in overweight people compared with people with normal BMI, which is consistent with studies in China ${ }^{20}$ and abroad. ${ }^{21}$ On the other hand, a Chinese study ${ }^{22}$ reported that individuals with low BMI were more prone to DR, which was also confirmed in other Asian countries. ${ }^{23}$ The above studies were cross-sectional surveys, while some Western cohort studies indicated that high BMI was associated with the progression of $\mathrm{DR},{ }^{24}$ and a Korean study also confirmed that weight reduction strategies could reduce the occurrence of DR. ${ }^{25}$ The discrepancies may be due to differences in study design and in the study population. Studies showing no association between BMI and DR could suffer from a reverse causality/survival bias. In addition, a Chinese study suggested that the relationship between BMI and DR prevalence was actually a U-shaped distribution. ${ }^{26}$ The association between BMI and DR needs further study using larger sample size.

Although some studies indicated that serum creatinine was an independent risk factor for DR, ${ }^{11}$ the results of this study did not reveal any association between serum creatinine and DR. After converting the serum creatinine values into CCR, it was found that the prevalence of DR increased with decreasing CCR, and that CCR was an independent risk factor for DR, which is supported by previous studies. ${ }^{27}$ In addition, the severity of DR is related to decreased glomerular filtration rate. ${ }^{27}$

The present study suggested that blood lipids were not independently associated with DR, which is supported by previous studies, ${ }^{20}$ but a previous Chinese study showed that hyperlipidaemia ( $\mathrm{TC} \geq 6.2 \mathrm{mmol} / \mathrm{L}$ ), very low-density lipoprotein cholesterol and TG were independent risk factors for $\mathrm{DR},{ }^{28}$ and a study confirmed the correlation between DR and TC and TG. ${ }^{21}$ American studies found that the occurrence of hard infiltration in the population with high TC and LDL was twice as much as that in the normal population, suggesting that TC and LDL are associated with the increasing risk of hard infiltration in the fundus. ${ }^{21}$ Therefore, further studies are needed for the relationship between blood lipids and DR.

The growth of the prevalence of diabetes in developing countries is higher than that in developed countries. ${ }^{1}$ In China, as a developing country with a large population, the prevalence of diabetes is steadily increasing and diabetes is diagnosed at a younger age. ${ }^{1}$ The present study was mainly conducted in young people (individuals $<60$ years of age accounted for $85 \%$ of the study population), and it was found that the prevalence of diabetes and prevalence of DR had the most important growth between 30 and 60 years of age. It can be seen from this study that although the prevalence of DR was low in the Chinese people, the frequency of diagnosis of diabetes and the prevalence of DR were significantly increased after 30 years of age. Therefore, screening programmes for diabetes in the general population and for DR in the population of patients with diabetes should be implemented, especially in individuals who have risk factors for diabetes and diabetic complications (eg, overweight, obesity, high blood pressure, dyslipidaemia and high FPG).

The present study has some strengths. The sample size was large. Fundus examinations were performed by experienced ophthalmic technicians and ophthalmologists. Screening of diabetes in the natural population was determined by OGTT. Nevertheless, there were some limitations. First, there is a possibility of a selection 
bias because the recruited individuals were visiting the hospital for a routine health examination. Second, not all subjects underwent an OGTT, which could underestimate the prevalence of diabetes. Third, Vujosevic et $a l^{29}$ showed that one-field examination does not necessarily reliably estimate the severity of retinopathy when compared with seven-field examination. Fourth, some individuals were excluded because of other eye diseases. Finally, fundus examinations without mydriasis could result in inaccurate diagnosis of DR. The selection criteria and the limitations of the study could limit the generalisability of the study. Nevertheless, the study population was from what could be considered the general Beijing population. Since the Beijing population is composed of Han people as well as minorities from the country, it could be considered as representative of the Chinese population.

In conclusion, we found that the prevalence of DR was $8.1 \%$ among patients with diabetes in our study population. Disease duration, BMI, SBP, CCR, UA and FPG were independently associated with DR.

Acknowledgements We acknowledge the Diabetes Prevention and Control Bureau of Beijing, the Center for Disease Control of Changping area and the Ophthalmic Research Institute of Beijing Tongren Hospital for their assistance in completing this project.

Contributors JC and JKY contributed to conception and design; JC, JPR, DNC, ZX, MXY, JX, QSY and JKY contributed to acquisition of data, or analysis and interpretation of data; JC, JPR, DNC, ZX, MXY, JX, QSY and JKY were involved in drafting the manuscript or revising it critically for important intellectual content; all authors gave final approval of the version to be published.

Funding This work was supported by grants from the National Natural Science Foundation of China (8151101058) and Beijing Municipal Science and Technology Project (D171100002817005) to JKY.

Competing interests None declared.

Patient consent Obtained.

Ethics approval Ethics committee of the Tongren Hospital.

Provenance and peer review Not commissioned; externally peer reviewed.

Data sharing statement Technical appendix, statistical code and dataset are available from the corresponding author.

Open Access This is an Open Access article distributed in accordance with the Creative Commons Attribution Non Commercial (CC BY-NC 4.0) license, which permits others to distribute, remix, adapt, build upon this work non-commercially, and license their derivative works on different terms, provided the original work is properly cited and the use is non-commercial. See: http://creativecommons.org/ licenses/by-nc/4.0/

(C) Article author(s) (or their employer(s) unless otherwise stated in the text of the article) 2017. All rights reserved. No commercial use is permitted unless otherwise expressly granted.

\section{REFERENCES}

1. Prokofyeva E, Zrenner E. Epidemiology of major eye diseases leading to blindness in Europe: a literature review. Ophthalmic Res 2012;47:171-88.

2. Yau JW, Rogers SL, Kawasaki R, et al. Global prevalence and major risk factors of diabetic retinopathy. Diabetes Care 2012;35:556-64.

3. Liu L, Wu X, Liu L, et al. Prevalence of diabetic retinopathy in mainland China: a meta-analysis. PLoS One 2012;7:e45264.

4. Report of the Expert Committee on the diagnosis and classification of Diabetes Mellitus. Diabetes Care 1997;20:1183-97.

5. Olafsdottir E, Andersson DK, Dedorsson I, et al. The prevalence of retinopathy in subjects with and without type 2 diabetes mellitus. Acta Ophthalmol 2014:92:133-7.
6. Zhang X, Saaddine JB, Chou CF, et al. Prevalence of diabetic retinopathy in the United States, 2005-2008. JAMA 2010;304:649-56.

7. Gunnlaugsdottir E, Halldorsdottir S, Klein R, et al. Retinopathy in old persons with and without diabetes mellitus: the Age, Gene/ Environment Susceptibility-Reykjavik Study (AGES-R). Diabetologia 2012:55:671-80.

8. Burgess PI, MacCormick IJ, Harding SP, et al. Epidemiology of diabetic retinopathy and maculopathy in Africa: a systematic review. Diabet Med 2013;30:399-412.

9. Thomas RL, Distiller L, Luzio SD, et al. Ethnic differences in the prevalence of diabetic retinopathy in persons with diabetes when first presenting at a diabetes clinic in South Africa. Diabetes Care 2013;36:336-41.

10. Raymond NT, Varadhan L, Reynold DR, et al. Higher prevalence of retinopathy in diabetic patients of South Asian ethnicity compared with white Europeans in the community: a cross-sectional study. Diabetes Care 2009;32:410-5.

11. Chiang PP, Lamoureux EL, Cheung $\mathrm{CY}$, et al. Racial differences in the prevalence of diabetes but not diabetic retinopathy in a multi-ethnic Asian population. Invest Ophthalmol Vis Sci 2011;52:7586-92.

12. Akhter A, Fatema K, Ahmed SF, et al. Prevalence and associated risk indicators of retinopathy in a rural Bangladeshi population with and without diabetes. Ophthalmic Epidemiol 2013;20:220-7.

13. Gadkari SS, Maskati QB, Nayak BK. Prevalence of diabetic retinopathy in India: the all India Ophthalmological Society Diabetic Retinopathy Eye Screening Study 2014. Indian J Ophthalmol 2016;64:38-44.

14. Wong TY, Cheung N, Tay WT, et al. Prevalence and risk factors for diabetic retinopathy: the Singapore Malay Eye Study. Ophthalmology 2008:115:1869-75

15. Hu HY, Lu B, Zhang ZY, et al. [An epidemiological study on diabetic retinopathy among type 2 diabetic patients in Shanghai]. Zhonghua Liu Xing Bing Xue Za Zhi 2007;28:838-40.

16. Xie XW, Xu L, Jonas JB, et al. Prevalence of diabetic retinopathy among subjects with known diabetes in China: the Beijing Eye Study. Eur J Ophthalmol 2009;19:91-9.

17. Wang FH, Liang YB, Peng XY, et al. Risk factors for diabetic retinopathy in a rural Chinese population with type 2 diabetes: the Handan Eye Study. Acta Ophthalmol 2011;89:e336-43.

18. Najam SS, Sun J, Zhang J, et al. Serum total bilirubin levels and prevalence of diabetic retinopathy in a Chinese population. $J$ Diabetes 2014;6:221-7.

19. Xu J, Wei WB, Yuan MX, et al. Prevalence and risk factors for diabetic retinopathy: the Beijing Communities Diabetes Study 6. Retina 2012;32:322-9.

20. Kunzmann S, Collins JJ, Kuypers E, et al. Thrown off balance: the effect of antenatal inflammation on the developing lung and immune system. Am J Obstet Gynecol 2013;208:429-37.

21. Liu L, Wu J, Yue S, et al. Incidence density and risk factors of Diabetic Retinopathy within type 2 Diabetes: a Five-Year Cohort Study in China (Report 1). Int J Environ Res Public Health 2015;12:7899-909.

22. Kung K, Chow KM, Hui EM, et al. Prevalence of complications among Chinese diabetic patients in urban primary care clinics: a cross-sectional study. BMC Fam Pract 2014;15:8.

23. Rooney D, Lye WK, Tan G, et al. Body mass index and retinopathy in Asian populations with diabetes mellitus. Acta Diabetol 2015;52:73-80.

24. Hammes HP, Welp R, Kempe HP, et al. Risk factors for Retinopathy and DME in type 2 Diabetes-Results from the German/Austrian DPV database. PLoS One 2015;10:e0132492.

25. Lim S, Kim KM, Kim MJ, et al. The association of maximum body weight on the development of type 2 diabetes and microvascular complications: MAXWEL study. PLoS One 2013;8:e80525.

26. Lu J, Hou X, Zhang L, et al. Association between body mass index and diabetic retinopathy in Chinese patients with type 2 diabetes. Acta Diabetol 2015;52:701-8.

27. Man RE, Sasongko MB, Wang JJ, et al. The Association of estimated Glomerular Filtration Rate with Diabetic Retinopathy and Macular Edema. Invest Ophthalmol Vis Sci 2015;56:4810-6.

28. Zhang HY, Wang JY, Ying GS, et al. Serum lipids and other risk factors for diabetic retinopathy in Chinese type 2 diabetic patients. $J$ Zhejiang Univ Sci B 2013;14:392-9.

29. Vujosevic S, Benetti E, Massignan F, et al. Screening for diabetic retinopathy: 1 and 3 nonmydriatic 45 -degree digital fundus photographs vs 7 standard early treatment diabetic retinopathy study fields. Am J Ophthalmol 2009;148:111-8. 